\title{
Toxicity Evaluation of Stearamidopropyl Dimethylamine Surfactant on Embryonic Development of Zebrafish \\ Hadeel Al-Jighefee ${ }^{1}$, Roba Abdin ${ }^{1}$ \\ Supervised by: Dr. Gheyath Khalid Nasrallah ${ }^{1,2}$, Ola Al-Jammal ${ }^{2}$ \\ ${ }_{1}^{1}$ Biomedical Science Department, College of Health Sciences, Qatar University, Doha, Qatar. ${ }^{2}$ Biomedical Research Center (BRC), Qatar University, Doha, Qatar
}

\section{ABSTARCT}

Background: Surfactants best known for their use in the industry of detergents, household products, and cosmetics. Usually, the amount of released surfactants into the environment is diluted during secondary water treatment. However, there is always a risk of releasing untreated and polluted wastewater containing high amounts of surfactants without knowing the harmful effect on biotic and abiotic elements of the environment. SAPDMA is a surfactant that is used mostly in cosmetics, conditioning agents, and recently in corrosion inhibition. The classification of SAPDMA as a "safe" or "green" surfactant by different environment or chemical agencies is ambiguous, and the literature is lacking.

Aim: The aim of this study is to evaluate the safety of SAPDMA using the zebrafish embryo model.

Methods: Zebrafish embryos were exposed to different concentrations of SAPDMA and the effect was assessed by different toxicity assays. This includes (i) mortality/ survival assay to assess the median lethal dose $\left(\mathrm{LC}_{50}\right)$ (ii) teratogenicity assay to assess the no observed effect concentration (NOEC); and (iii) organ specific toxicity assays including cardiotoxicity, neurotoxicity (using locomotion assay), and hemoglobin synthesis (using odianisidine staining)

Results: Exposure of zebrafish embryos to SAPDMA caused mortality in a dose-dependent manner with a calculated $\mathrm{LC}_{50}$ of $2.3 \mathrm{mg} / \mathrm{L}$. Thus, based on the $\mathrm{LC}_{50}$ value and according to the Fish and Wildlife Service Acute Toxicity Rating Scale, SAPDMA is classified as "moderately toxic". The NOEC, the concentration that did not cause any significant teratogenicity, was $0.1 \mathrm{mg} / \mathrm{L}$. However, this concentration caused significant organ specific and cytotoxic effects, suggesting that harmless concentrations of SAPDMA are lower than $0.1 \mathrm{mg} / \mathrm{L}$. Conclusion: Our data indicate that SAPDMA at very low concentrations causes adverse effects on zebrafish embryos. Thus, we recommend that the use of SAPDMA in industry should be re-evaluated and monitored by the environment and public health agencies.

\section{INTRODUCTION}

Surfactants are widely used in the industry due to their significant physio-chemical properties. After use, residual surfactants are dispensed into sewage systems or into surface waters, most of which end up dispersed into different environmental pools. Most surfactants rapidly biodegrade, and their concentration is greatly reduced during secondary wastewater treatment. Thus, the major concern lies in the possibility of releasing untreated wastewater that is polluted with large amounts of surfactants. This could lead to serious effects on the ecosystem. Few studies have investigated the impact of surfactants on the aquatic fauna. SAPDMA is the most common cationic surfactant used in cosmetics, personal care products and recently in corrosion inhibition. According to the Environment Canada Domestic Substance List, SAPDMA is classified as a low hazardous ingredient and not expected to be toxic. However, other databases classified SAPDMA as a very toxic compound to the aquatic environment with long-lasting effects. There are no performed studies to assess the comprehensive toxicity and safety of SAPDMA on the ecosystem. Also, if for any reason this surfactant poses a toxic effect on the environment, it is inevitable that this effect will also reach and adversely affect humans through bioconcentration. In this study, zebrafish embryo model was used to, which are a great representative model for the ecosystem, to assess the safety of SAPDMA.

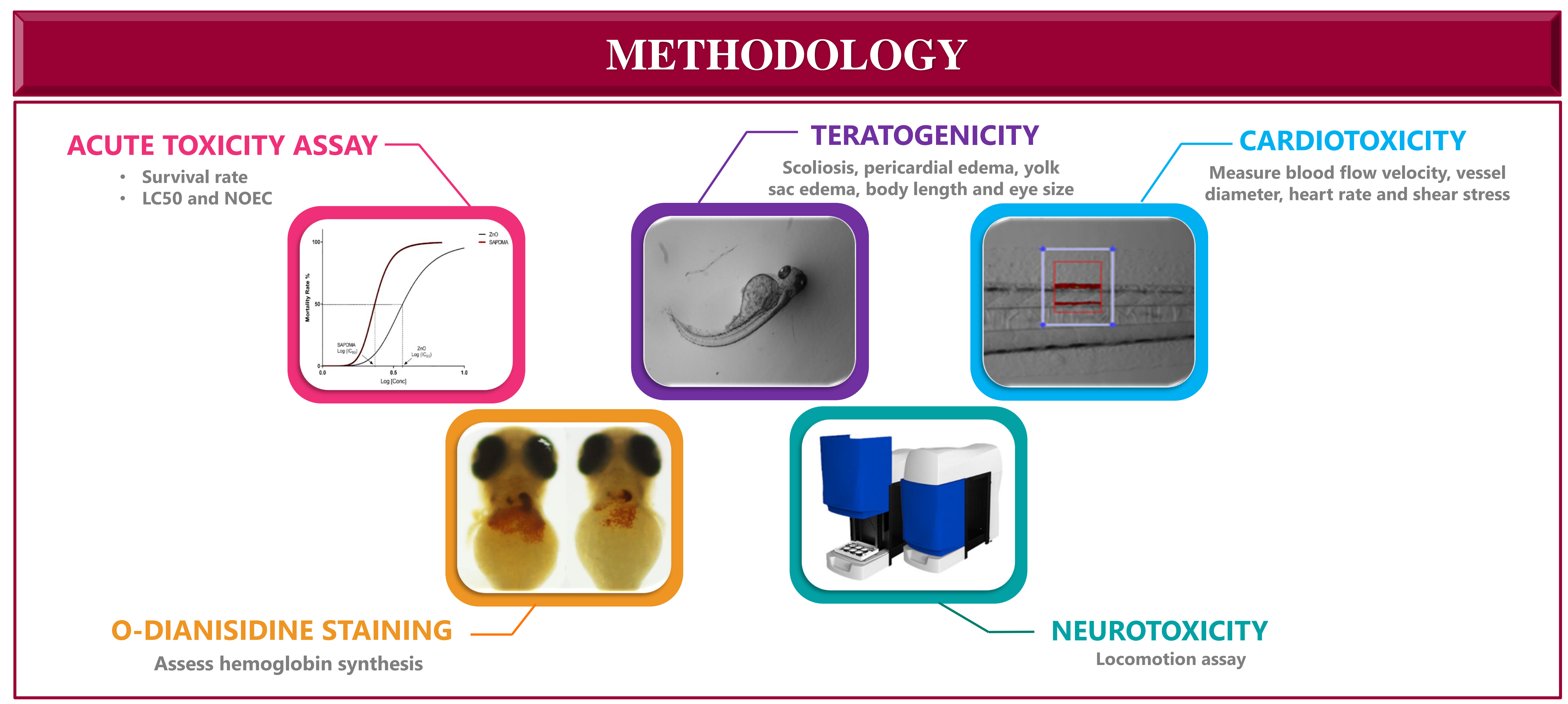

RESULTS
Table 1. Concentrations of SAPMDA at which the SAPDMA (mg/L)

\begin{tabular}{|cc|}
\hline LC $_{\mathbf{5 0}}$ & 2.3 \\
\hline NOEC & 0.1 \\
\hline $\mathbf{1 0 0 \%}$ mortality & 3.5 \\
\hline
\end{tabular}

$100 \%$ mortality

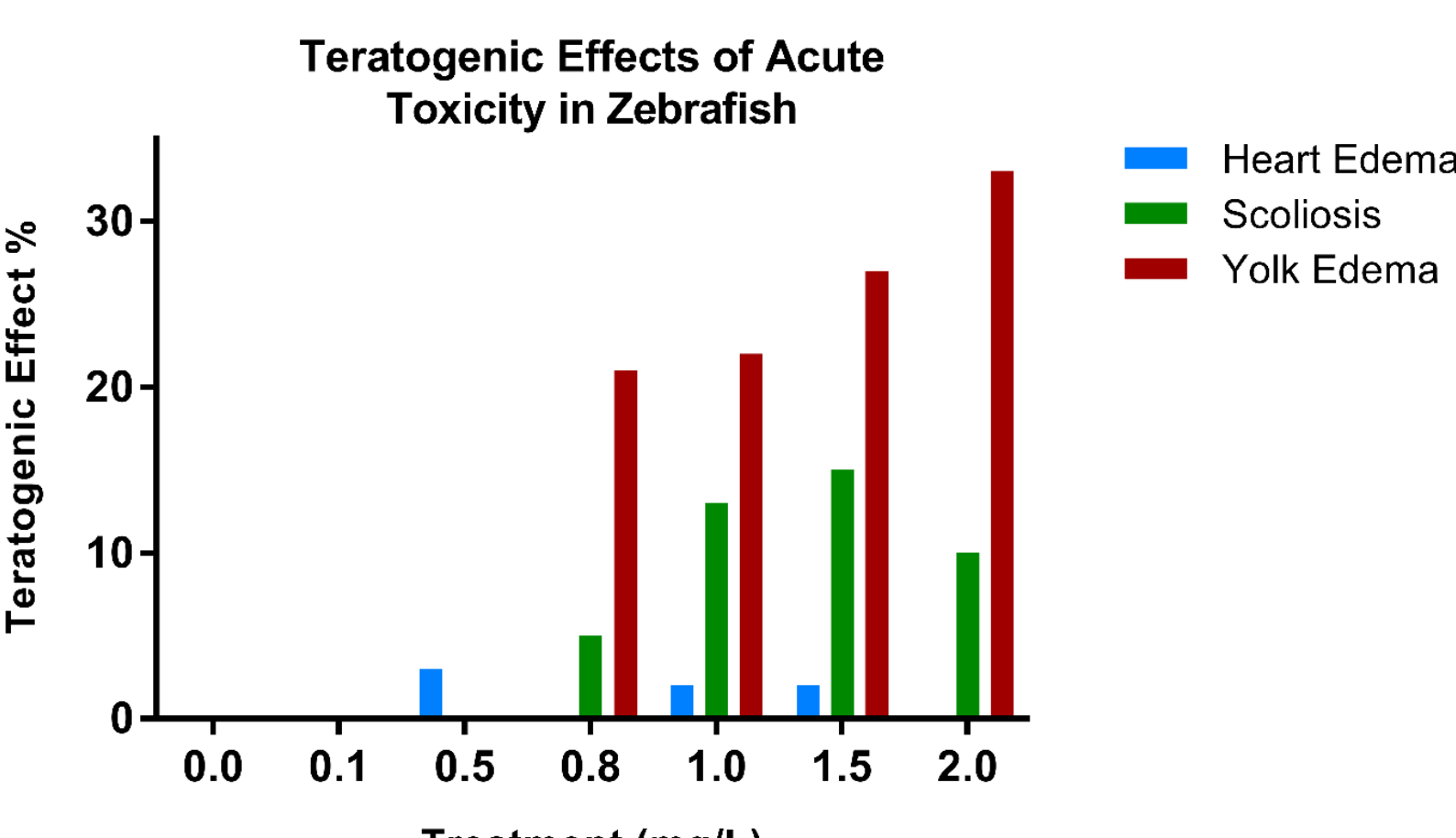

A)

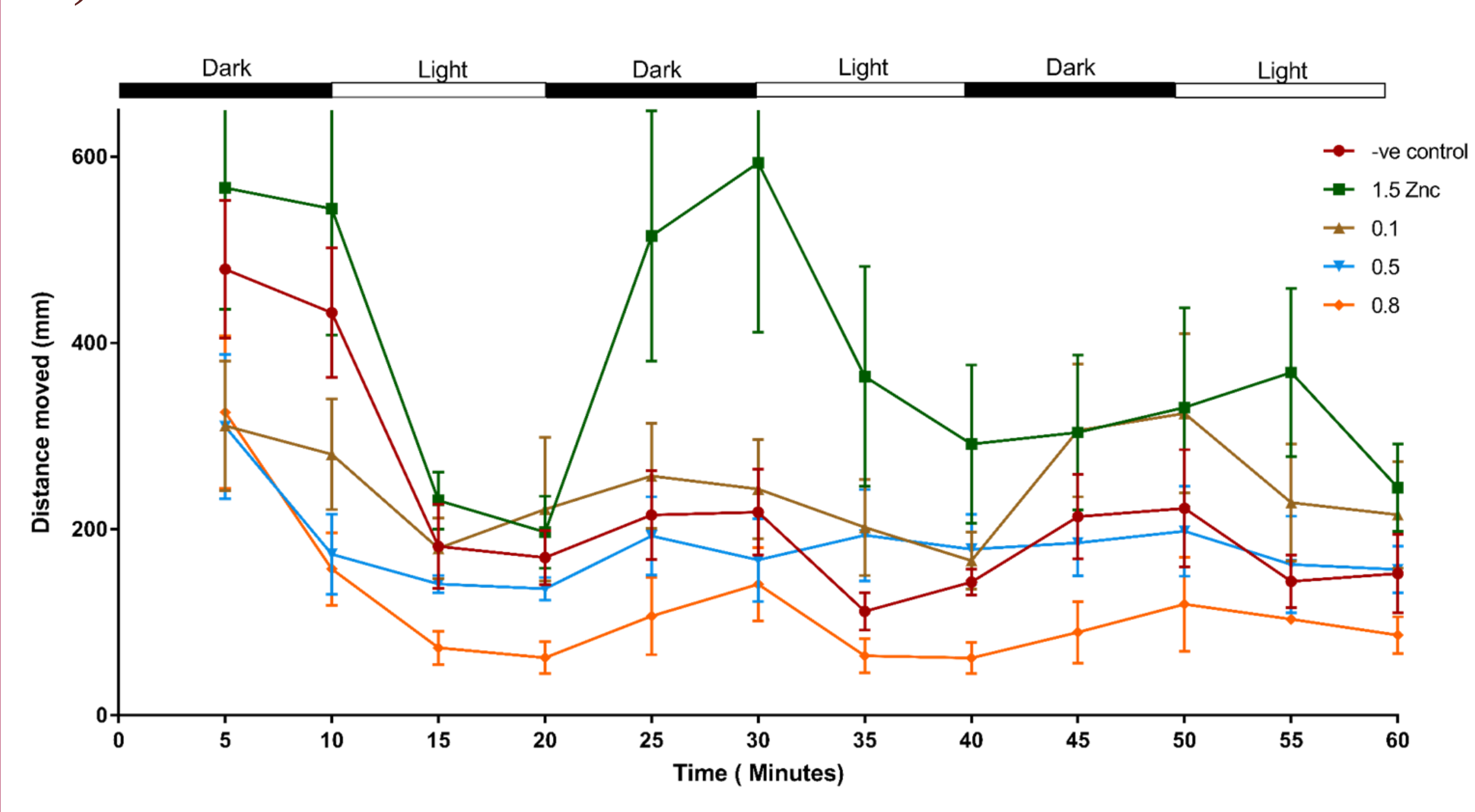

B)

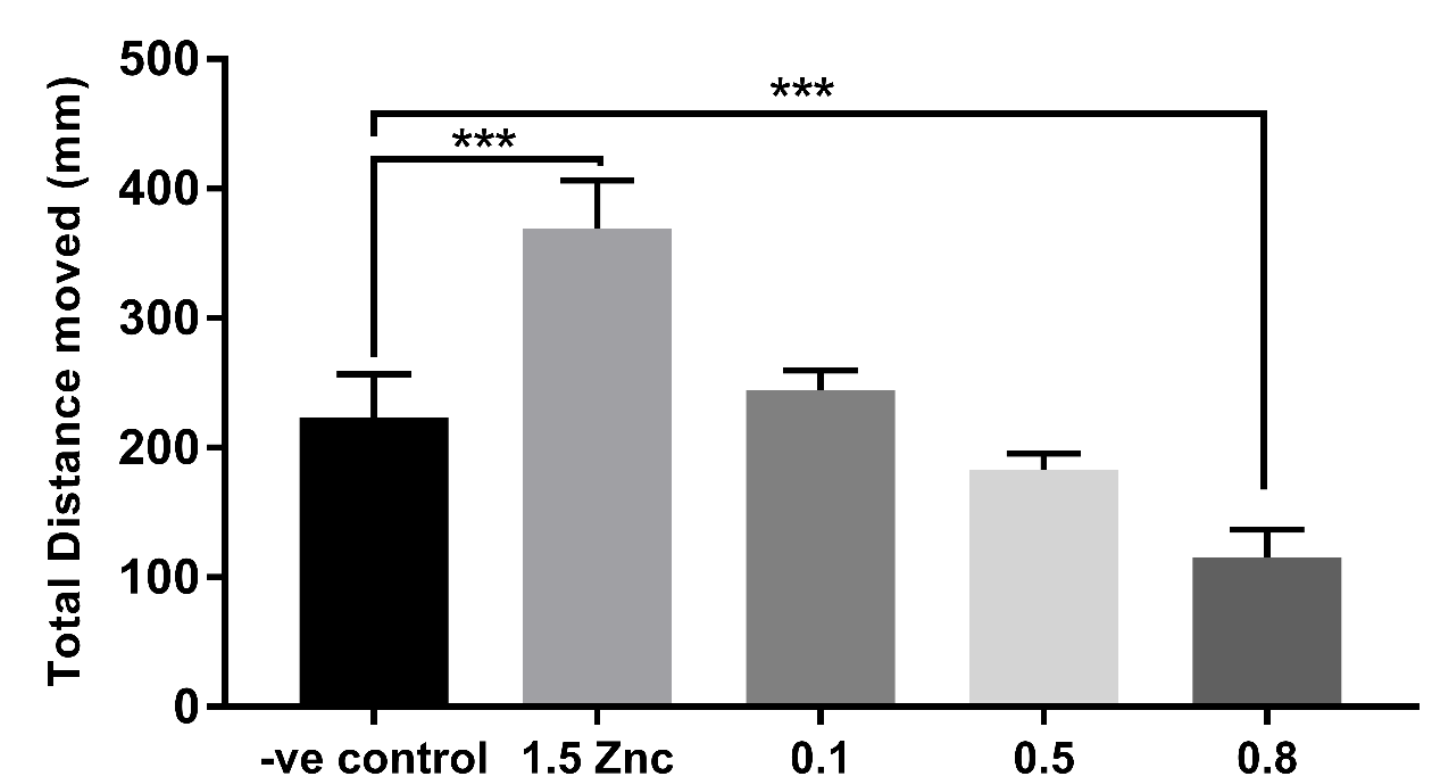

Figure 2. Normal locomotion of SAPDMA treated zebrafish embryos. (A) Average total distance (measured using ViewPoint, Micro lab system) moved
(in mm) by the 120-hpf- old embryos per minute treated at $96 \mathrm{hpf}$ with PTU, (in $\mathrm{mm}$ ) by the 120 -hpf- old embryos per minute treated at $96 \mathrm{hpf}$ with PTU,
Figure 1. Percentage of embryos with teratogenic moved (mm) per hour under dark/ light phases of the same embryos as in $(\mathrm{A})$.

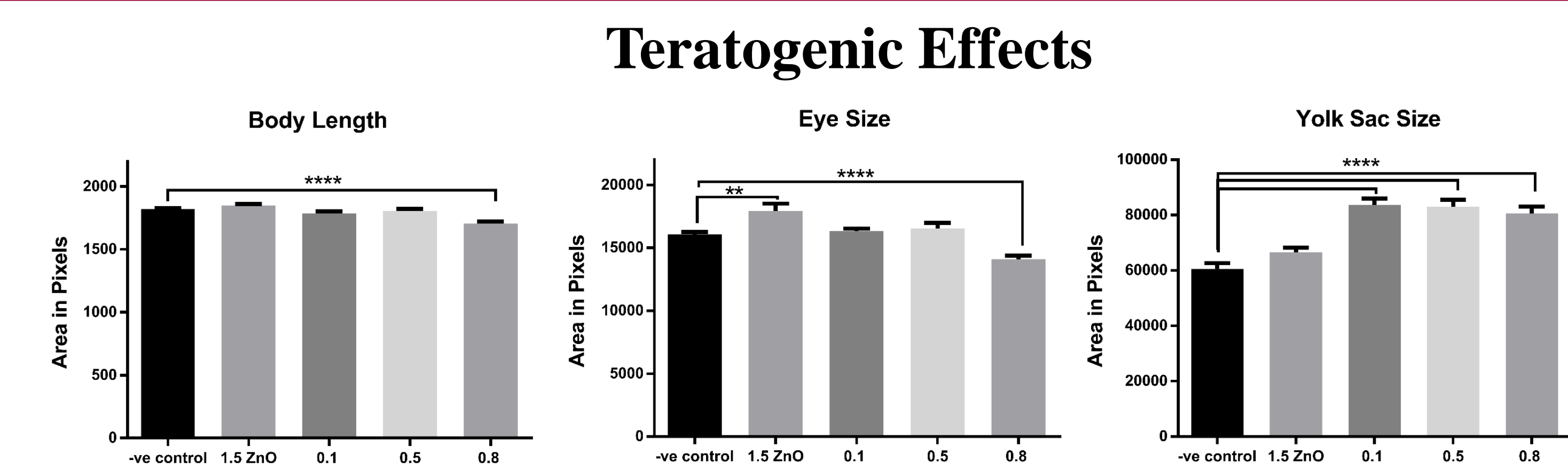

Figure 3. Teratogenic effects of different SAPDMA concentrations. Body length, eye and yolk sac size of the embryos following treatment, were measured using imageJ software. One-
way Anova was used to compare between the negative control and ach treated A) Cardiotoxicity

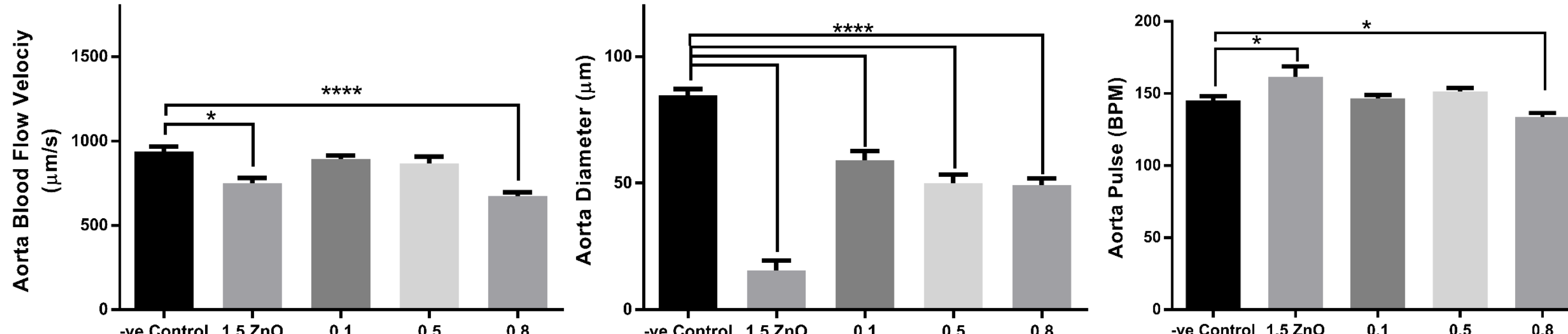
B)

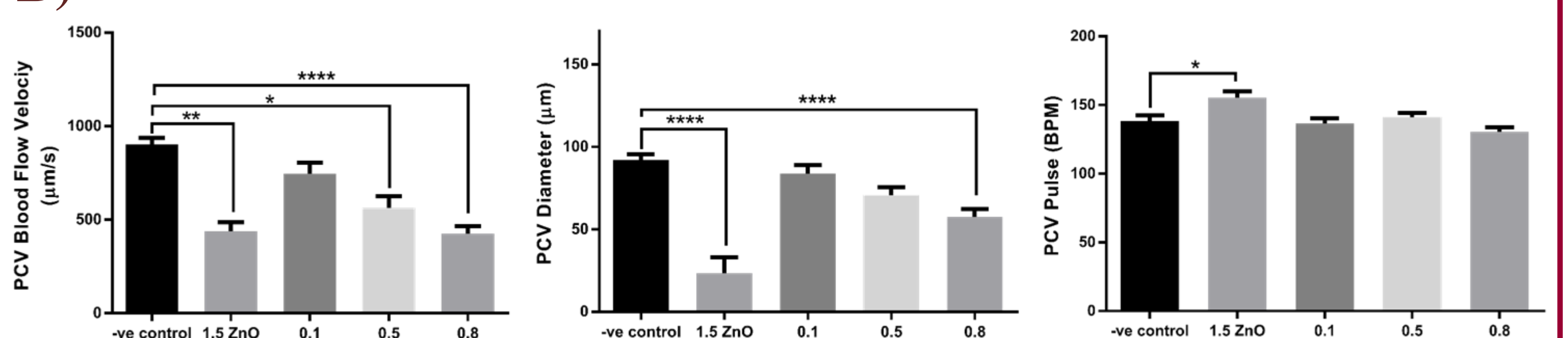

Figure 4. Effect of SAPDMA on blood flow velocity, diameter and heart rate. Parameters were calculated from the (A) dorsal aorta (DA) and (B) Posterior cardinal vein (PCV) of the embryos following treatment with each indicated concentration. Twenty embryos were used per concentration. One-way ANOVA was used to compare the differences between the a between groups. " $p<0.05$ and

Hemoglobin Synthesis

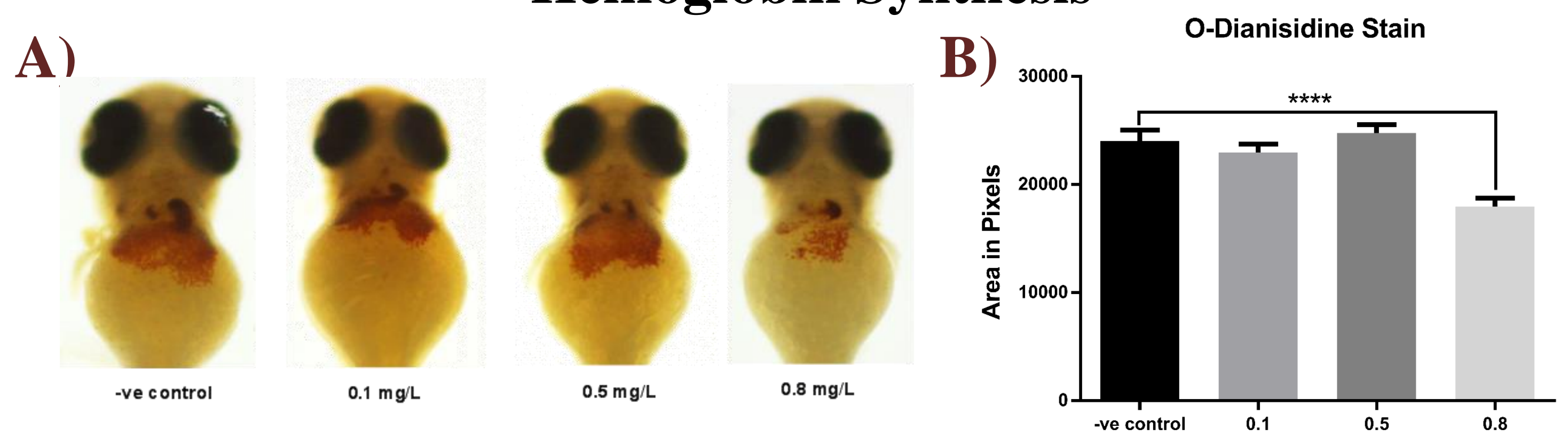

Figure 5. Distribution of hemoglobin-positive cells was detected by O-dianisidine staining at 72 hpf. (A) Representative images of o-dianisidine Stain concentrated on the yolk sac of the control, $\mathrm{ZnO}$ microscopy, at $50 \mathrm{X}$. (B) The graph demonstrates the difference in the number of erythrocytes with egards to difference in the areas stained by o-dianisidine. No significant difference was observed between the control, 0.1 and 0.5 SAPDMA $(\mathrm{mg} / \mathrm{L})$. However, a significant difference $(\mathrm{P}<0.0001)$ was observed between the control and 0.8 SAPDMA $(\mathrm{mg} / \mathrm{L})$. ***** $p<0.0001, n=25$

\section{CONCLUSION}

All performed toxicity assays strongly suggest that SAPDMA has a potential toxic effect on the organ development of zebrafish embryos in a dose-dependent manner. To our knowledge, no previous studies were done to evaluate the toxicity of SAPDMA and the present work is the first to report a full toxicity assessment of this surfactant on zebrafish model. We concluded that SAPDMA is classified as a "moderately toxic" compound according to the FWS acute toxicity scale. Thus, the concentrations of SAPDMA used in the industry and present in the environment should be monitored. Also, further studies are warranted to determine the long-term toxicity of SAPDMA.

\section{REFERENCES}

Radwan, A., Sliem, M., Okonkwo, P., Shibl, M., \& Abdullah, A. (2017). Corrosion inhibition of API X120 steel in a highly aggressive medium using stearamidopropyl dimethylamine. Journal of Molecular Liquids, 236, 220-231.

Vaughan, M., van Egmond, R. (2010). The Use of the Zebrafish (Danio rerio) Embryo for the Acute Toxicity Testing of Surfactants, as a Possible Alternative to the Acute Fish Test. Alternatives to laboratory animals: ATLA. 38. 231-8

Wang, Y, Zhang, Y, Li, X, Sun, M, Wei, Z Wang, Y, Gao, A, Chen, D, Zhao, X \& \& Feng, X (2015). Exploring the Effects of Different Types of Surfactants on Zebrafish Embryos and Larvae. Scientific reports. 